\title{
Interactive comment on "Evaluating a fire smoke simulation algorithm in the National Air Quality Forecast Capability (NAQFC) by using multiple observation data sets during the Southeast Nexus (SENEX) field campaign" by Li Pan et al.
}

Anonymous Referee \#1

Received and published: 19 January 2019

Re- review: Li Pan et al.

Journal: GMD Title: Evaluating a fire smoke simulation algorithm in the National Air Quality Forecast Capability (NAQFC) by using multiple observation data sets during the Southeast Nexus (SENEX) field campaign Author(s): Li Pan et al. MS No.: gmd2017-207 MS Type: Model evaluation paper 
and air quality modeling system, with a particular effort to utilize the SENEX campaign observations. Their motivation is apparently to provide guidance on use or further development of the very similar NAQFC system, as cited in lines 44-45 in the introduction: '....National Air Quality Forecasting Capability (NAQFC) daily PM2.5 operational forecast (Lee et al., 2017).'

The authors describe numerous analyses they completed to compare the 'fire signals' to be found in the CMAQ model results: deltaCO from CMAQ, PM2.5 CO and EC, acetonitrile, AOD, satellite fire hotspot detects and plume extents.

Since the NAQFC is explicitly cited as being for the purpose of predicting PM2.5, and since this paper seeks to evaluate a NAQFC analogue, it seems quite odd that there was no effort to compare the SENEX EC and OC PM2.5 with model results, except in terms of ratios. While the paper shows considerable and diverse efforts to utilize appropriate data to evaluate the simulation results, poor writing obscures the value and meaning of this work to an unacceptable extent. The paper is authored by a respectable set of scientists; it is hard to believe that most of these authors actually read the paper as reviewed, so rife was it with grammatical errors, confusing word choices, contorted syntax.

specific comments

I saw no effort to directly compare CMAQ PM2.5 with SENEX PM2.5 EC and OC.

Figures $7 \mathrm{a}, \mathrm{b}, \mathrm{d}$ and e are all missing color bar legends for AOD.

In Figures $8 \mathrm{a}$ and $\mathrm{b}$ and $9 \mathrm{e}$, the square symbols for observations are so densely packed that their outlines (in black) obscure the symbol colors over much of the flight path. Perhaps the density of observation points could be reduced in some areas and/or the symbols made larger to address this.

technical corrections Before submission for review, it is hoped that all the authors of the paper have read and edited the MS. I very much doubt that was the true here, as this

Printer-friendly version

Discussion paper 
MS is rife with grammatical errors and confusing constructions. I could not offer this to students as a model of scientific writing. Some of these are indicated below, but really, there are so many that I didn't get to all of them.

Line 29, comma after campaign Line 33 change 'helped identified' to showed or identified Line 36 , change 'filter out' to retrieve or to 'focus on' Line 77 change 'comprised' to consists to make this a sentence. Change Satellite to Satellites Line 82 and following: itemization of the file names could maybe be best isolated in supplemental material. It isn't clear that using file names in this discussion adds much. Line 94: is hmx.txt meant to read hms.txt as used above. Line 97: HMS imagery is Line 109 and elsewhere: In remote sensing a 12-km grid does not reliably 'resolve' features of size of $12 \mathrm{~km}$, so I object to this casual misuse of 'resolution', even though it is common. Say '12- km grid' or otherwise describe. Use 12-km as adjective for grid. Be consistent. Line 121-125: Confusing Line 128: emission rates Line 132: gridded emission Line 143: If that's a crude estimate what is a better approach and why wasn't that tested? Line 166-168: confusing. Reword. Line 171: is emitted by biomass burning Line 181-183 Not sure, but this sounds like you intend to tell us which processes contribute how much error. Line 188-190: But apparently not... Line 190: the purpose is to focus on fire/smoke signal timing? Line 205: Table 1 only gives AGL, not ASL. Lines 210 and 214: change exhibits to shows Line 219 - 222: Unclear Line 222-225: Not a sentence, even. Line 227: 'not negligible perspective' is unclear Line 246: change 'below' to 'above' and change 'was' to 'were' Line 252: Change Tab. to Table Line 269: Change 'for' to 'to' Line 284 - 292: Could you say something clarifying about the significance of interference from clouds in making informative FMS comparisons? Line 290: CMAQ didn't underestimate it, the HMS BlueSky SMOKE emissions system did. Line 294-5. No, your system used a climatological LBC and was thus blind to whether there was more or less actual influence from external fires. Line 303: 'a similar analysis' or 'similar analyses'. Line 302: 'is accessed' Line 309: 'Other reasons. .. are discussed. . ' Line 331: change sparingly to occasionally or rarely. Line 334: change that to those or change that to were. Line 338: change 'are subject' to tend Line 387: So $\mathrm{CH} 3 \mathrm{CN}$ decreased

Printer-friendly version

Discussion paper 
along with AGL, as AGL decreased? Or was inversely related to AGL? An ambiguously statement as written. Line 398: change 'was' to 'were' Line 403-5: The single isolated $\mathrm{CH} 3 \mathrm{CN}$ value of $3000+$ strongly affects the slopes in Figures $9 \mathrm{c}$ and $9 \mathrm{~d}$. Line 444: 'rely on predicted delta $\mathrm{CO}$, the difference. ...' Line 449: delete 'similar', change 'compared with' to 'comparable to' Line 450: change 'shapefile analysis' to 'shapefiles' Line 452: end sentence as 'from elsewhere in the CONUS domain.' Line 457: change 'outside' to 'bounding' Line $461-471$ : For a structure like this with a colon leading to a list of independent clauses (1-4) (that may or may not contain commas), begin each clause in lower case and terminate all the clauses with a semicolon. Except for the last one, which gets a period. Line 463-4: 'to avoid impasse arose by uncertainties' is unclear 468-9: we were intentionally conservative... Line 470: 'outliers' and delete 'sparse' Format used in text for citation is inconsistent.

Interactive comment on Geosci. Model Dev. Discuss., https://doi.org/10.5194/gmd-2018-230, 2018. 